\title{
PO3-1-7
}

Poster session

\section{Common blood-based markers in female patients and a model mouse of depression}

\author{
Shigeo Miyata ${ }^{1,2}$, Masahiko Mikuni $^{2}$, Yuchio Yanagawa' ${ }^{1}$, Masato Fukuda ${ }^{2}$ \\ ${ }^{1}$ Dept. of Genetic and Behavioral Neuroscience, Gunma University, Japan, ${ }^{2}$ Dept. of Psychiatry and Neuroscience, \\ Gunma University, Japan
}

Background: To discover novel antidepressant agents, animal models of depression are useful for pharmacological studies. The depression-like state of these animals is generally evaluated by their behaviors; however, these behavioral indices have substantial limitations for drug discovery because of their weak validation in human major depressive disorder (MDD). The aim of this study was to identify the objective markers in common between patients and a model mouse of depression among the transcripts in blood cells.

Methods: In the clinical study, the blood was freshly obtained from the female patients with MDD (age > 50 years) who were receiving treatment at the Department of Psychiatry and Neuroscience at Gunma University Hospital. The blood of age- and gender-matched healthy individuals were also obtained. Total RNA of white blood cells was extracted, and the gene expression in each sample was analyzed by the microarray. The differentially expressed genes and the biological pathways were evaluated and compared with those in the mouse model of depression (bilaterally ovariectomized mice) which has already been reported by us (Miyata et al., Heliyon, 2016). All the participants received complete information on this study and signed an informed consent document.

Results: The female patients with depression $(n=10)$ showed the up-regulated 337 genes and the down-regulated 78 genes in the blood cells compared with the healthy individuals $(n=7)$. The up-regulated genes were mainly associated with oxidative phosphorylation and mitochondrial dysfunction, which were consistent with the mouse model of depression.

Conclusion: We identified the biological alteration associated with oxidative phosphorylation and mitochondrial dysfunction in the blood cells of female patients with MDD. Interestingly to note that these biological alterations were also found in the blood cells of mouse model of depression (Miyata et al., Heliyon, 2016). Therefore, it is possible that the blood-based marker is more suitable for evaluating the depression-like state of model animals of depression than the behavioral indices. 